Le secret médical est comme une montre à complications: c'est sa complexité qui fait sa richesse, mais son application suscite beaucoup de questions. Le discours inquiet sur la dangerosité de certains détenus et la prétendue cécité des médecins a mis sous pression les psychiatres forensiques. Les divergences apparues ne sont pourtant pas liées à un Röstigraben, comme le montre l'article ci-dessous, car la loi est claire, mais à la crainte des médecins à utiliser pleinement toutes les potentialités du secret médical, finalement à l'avantage de la société.

\title{
Le secret médical en prison: une fausse polémique
}

\author{
Dominique Sprumont ${ }^{a}$, Philippe Ducor ${ }^{b}$ \\ a Prof. Dr iur., Directeur adjoint de I'Institut de droit de la santé, Vice-directeur SSPH+ / ERSP, Université de Neuchâtel
}

${ }^{b}$ Prof. Dr iur., Dr med., avocat, Genève

Sous le titre Mythen und Realitäten, Steffen Lau et Josef Sachs ont récemment revisité la question du secret médical dans la prise en charge de détenus dangereux [1]. Leur intention était de recentrer le débat sur la réalité de la psychiatrie forensique en réponse au discours "émotionnel» qui, selon eux, prédomine en Suisse romande. Malheureusement, loin de combattre les mythes, l'article ne fait que les accentuer tout en prenant quelques libertés avec le cadre légal de la pratique médicale en prison.

Premièrement, il convient de rappeler un principe juridique de base selon lequel en l'absence d'une règle de droit spécifique, on applique les règles générales. Concrètement, cela signifie que les médecins intervenant en prison ou mandatés par la justice sont tenus de respecter les mêmes règles fondamentales avec les patients détenus qu'avec les patients en liberté, à moins qu'il n'existe des dispositions légales particulières qui modifient leurs droits et obligations. Selon Lau et Sachs, le rapport de droit spécial entre le détenu dangereux et l'administration pénitentiaire et judiciaire serait un motif justificatif suffisant. Ils sous-entendent que, dès lors que la relation patient-médecin en prison ne relève pas du droit privé, le régime des droits des patients et des obligations professionnelles serait fondamentalement différent. Cet argument laisse songeur dans la mesure où, appliqué à la lettre, il impliquerait que les patients hospitalisés dans des établissements de droit public, non soumis au droit privé, seraient aussi placés sous un régime spécial. Il n'en est pourtant rien. Il suffit pour cela de se référer à l'art. 40 de la Loi fédérale sur les professions médicales (LPMéd), de même qu'aux nombreuses législations

cantonales sur la santé, qui imposent aux médecins la même obligation générale de respecter les droits des patients dans le secteur public et dans le secteur privé. Le médecin reste toujours médecin. Ce principe est du reste confirmé par les lois cantonales d'application du Code pénal qui reconnaissent qu'en matière de santé, les détenus ont les mêmes droits que les autres personnes. La FMH a ainsi toujours fermement défendu le principe de l'équivalence des soins en médecine pénitentiaire ou, encore récemment, dans le cadre des renvois forcés.

En l'absence d'une règle de droit spécifique, on applique les règles générales.

Lau et Sachs soutiennent aussi dans leur conclusion qu'il ne faut pas seulement tenir compte du droit des détenus au respect de leur sphère privée, mais également de celui de la société d'être protégée. Cet argument rappelle le slogan de certains politiciens lors des affaires Adeline et Marie, selon lequel «le doute doit bénéficier à la société». Posé en des termes aussi généraux, il est difficile de leur donner tort. La réalité est toutefois plus complexe. L'enjeu est moins dans les droits des détenus dangereux que dans le mandat que
1 Lau S, Sachs J. Mythen und Realitäten. Bull Méd Suisses. 2015;96(37):1331-3. 
la justice confie aux médecins en vertu du Code pénal (CPS). Pour mémoire, c'est justement parce qu'ils sont dangereux que les juges prescrivent des mesures thérapeutiques [2] dans le but de faire baisser leur dangerosité. C'est une obligation à laquelle le juge ne peut déroger. Juridiquement, le psychiatre forensique qui agit sous mandat de la justice a ainsi un véritable devoir vis-à-vis de la société, dans le respect de ses obligations de médecin. C'est précisément en raison de ce mandat que de nombreux médecins pénitentiaires, les sociétés cantonales de médecins, la FMH et l'Académie Suisse des Sciences Médicales ont défendu et continueront à défendre le secret médical en prison. La mission du psychiatre forensique est impossible sans secret.

\section{Cela ne signifie pas pour autant que médecin et autorités pénitentiaires et judiciaires ne peuvent pas communiquer.}

L'efficacité d'un traitement dépend dans une large mesure du rapport de confiance qui s'établit entre patient et médecin. Le secret médical [3] est la pierre angulaire de ce rapport de confiance. Le mettre à mal prive le thérapeute d'une partie de sa capacité de soigner, vidant ainsi de son sens le mandat thérapeutique prévu dans le Code pénal. Cela ne signifie pas pour autant que médecin et autorités pénitentiaires et judiciaires ne peuvent pas communiquer. Au contraire, comme le constatent avec justesse Lau et Sachs, toute mesure thérapeutique fait l'objet d'un suivi par l'autorité en charge de l'application des peines. Le "mandat thérapeutique» établi entre le patient-détenu, le médecin et la justice, en pleine connaissance et accord du patient-détenu, précise les éléments qui font l'objet d'un échange d'information entre le médecin et la justice, par exemple le respect des prescriptions médicales, la participation aux séances, l'avancement du traitement selon les objectifs de diminution de la dangerosité, etc. Les rapports doivent être fournis de manière régulière sur la base d'un calendrier préétabli. Ces éléments ne sont pas contestés et font partie intégrante de la pratique médicale en prison en Suisse.

La question soulevée par la révision de certaines lois d'application du Code pénal en Suisse romande est ailleurs. Elle porte sur les faits et évènements survenant en dehors du mandat thérapeutique, qui pourraient avoir une incidence sur l'appréciation des risques présentés par le détenu. A ce propos, il convient de rappeler la différence fondamentale entre le rôle de thérapeute et celui d'expert que sont amenés à assumer les psychiatres forensiques. Le Code de procédure pénale
(CPP) établit une distinction claire entre les deux, un expert ne pouvant en même temps agir comme thérapeute et inversement [4]. Il s'agit d'un principe général du droit, qui se traduit par des règles de révocation également consacrées dans les codes de procédures civile [5] et administrative [6]. La raison en est simple. Le thérapeute se trouve dans un rapport de proximité avec son patient, ce qui est susceptible d'altérer son jugement et constitue une source de conflits d'intérêts potentiels. Afin de protéger la société, le Code pénal a dès lors établi des procédures spécifiques visant à évaluer la dangerosité des délinquants particulièrement à risque [7]. Cette évaluation se fait sous contrôle judiciaire et avec la participation de plusieurs experts, le plus souvent dans un regard interdisciplinaire. En ce qui concerne la transmission d'information par un médecin à la justice dans le cadre d'une procédure pénale, il convient encore de rappeler l'art. 171 al. 3 CPP qui réserve explicitement le secret médical. Le Tribunal fédéral a ainsi jugé contraire à cette disposition la loi sur la santé bâloise qui entendait créer un devoir général d'information dans de telles circonstances [8].

Il est regrettable que Lau et Sachs ne tiennent pas mieux compte de la différence entre le rôle de thérapeute et celui d'expert. Leur article donne à penser que les médecins qui remplissent consciencieusement leur rôle de thérapeute manquent à leur devoir vis-à-vis de la société. Ils semblent défendre l'idée que les médecins en prison devraient être des auxiliaires de la justice avant d'être des thérapeutes. Cela est inquiétant, car contraire aux objectifs même du Code pénal et de la politique criminelle qu'il consacre. Dans leur caricature des médecins soucieux du secret médical en prison, prétendument trop proches de leurs patients, Lau et Sachs omettent de mentionner qu'en pratique cesmêmes médecins communiquent régulièrement avec la justice et les autorités pénitentiaires sur la base de leur mandat thérapeutique. De même, ils feignent d'ignorer que, conformément à l'article 17 CPS, il arrive à ces mêmes médecins d'informer sans tarder les autorités d'un danger imminent.

Il n'y a pas en médecine une seule vérité. Il est sain que les praticiens puissent défendre des approches différentes. Le cadre légal reste toutefois le même pour tous, et il n'est pas inutile d'en rappeler la teneur. La FMH et la Conférence des Médecins Pénitentiaires Suisses (CMPS) sont bien placées pour le faire! A partir de là, il sera possible aux spécialistes de se positionner en identifiant les pratiques acceptables, plutôt que de se perdre dans de fausses polémiques. 